\title{
Analysis of the evolution of road tunnels equilibrium conditions with a convergence-confinement approach
}

\author{
Federica Sandrone - Vincent Labiouse
}

Received: 8 December 2008/Accepted: 31 May 2009/Published online: 18 June 2009

(C) Springer-Verlag 2009

\begin{abstract}
Since both lining structure and rock mass exhibit delayed behaviour, tunnel equilibrium conditions evolve with time. After discussing existing work done on different aspects of long-term tunnel behaviour, the aim of this paper is to "understand" the influence of rock mass and lining degradations on the long-term stability conditions of the tunnel by means of the convergence-confinement method. In order to represent the effects of degradation on tunnel long-term conditions, specific degradation models are selected according to the disorders identified during principal inspections of road tunnels in Switzerland. By simulating the reduction of the mechanical properties of both the rock mass and the lining or by using creep models, it is possible to assess the influence of the main degradation processes on tunnel stability. The results are interpreted in terms of tunnel safety factor. The presented approach for the determination of the long-term behaviour of tunnels, although valid for simple tunnel geometry and field stress conditions, allows to roughly estimate the influence of significant degradation processes that affect the rock mass and the supporting structure. Though this approach results in some simplification, it may be generalised and adopted with more refined numerical analyses for improving the assessment of tunnel long-term conditions.
\end{abstract}

F. Sandrone $(\bowtie) \cdot$ V. Labiouse

Rock Mechanics Laboratory, LMR,

École Polytechnique Fédérale de Lausanne, EPFL,

Station 18, 1015 Lausanne, Switzerland

e-mail: federicalucia.sandrone@epfl.ch

URL: http://lmr.epfl.ch/
Keywords Road tunnels · Degradation · Long-term behaviour - Convergence-confinement method . Safety factor
Abbreviations
GCC Ground characteristic curve
SRL Support reaction line
FoS Factor of safety

\section{Introduction}

Tunnels, like other structures, evolve and their properties change with time. Indeed, after several years of operation, various degradation processes may affect tunnels, reducing their serviceability and safety levels. Currently, due to the age of the tunnels of the road networks, in all European countries, the major tasks for a tunnel engineer are management and conservation practices. This requires appropriate tools in order to:

- follow the life of tunnels from their construction and through their operation;

- understand the mechanisms that characterise the longterm degradation of tunnels and identify the main pathologies, their causes and their effects in terms of tunnel performance;

- improve maintenance techniques.

Due to its mountainous topography, the roads in Switzerland have a fairly large number of tunnels. Since the road network was developed a lot during the 1960s, today a significant number of the tunnels has already been in operation for more than 30 years and needs efficient conservation practices to ensure everyday safety and 
serviceability. For this reason, in the framework of a Ph.D. thesis financed by the Swiss Federal Roads Authority, the long-term behaviour of the National Road tunnels has been studied.

By considering the type of degradation process and its effects, it is possible to divide the tunnel pathologies that affect both the rock mass and the concrete lining into three main classes:

1. Ageing that groups all physical processes ruled by time-dependent changes affecting the internal characteristics of concrete and rock. These processes may be modelled by a stiffness decrease (or a strain increase).

2. Weathering which groups all chemical processes that affect exposed surfaces (e.g. lining intrados, weak zones and discontinuities). This kind of degradation is caused by the interaction of the tunnel with the surrounding environment. These processes mainly reduce material strength and mechanical properties. Also thickness reduction due to weathering of the exposed material can be observed.

3. Other actions, which include several mechanical processes such as pore water pressure redistribution, ice and frost action, fires and car collisions which may also change the tunnel equilibrium conditions with time.

Based on these considerations, by means of specific data collection on National Road tunnels (Sandrone et al. 2007; Sandrone 2008) and a detailed literature review it has been possible to identify for each of the main pathologies the respective symptoms (i.e. disorders, damages, defects) that can be observed during principal inspections, the affected zones and the potential influencing factors (i.e. causes). Moreover, the main pathologies have been described by simplified models, which take into account the main effects in terms of mechanical (or geometrical) properties of both the lining and the rock mass.

Based on the tunnel initial conditions and on the degradation rate of both the rock mass and the lining, it should be possible to assess the evolution with time of the stability conditions. Since the tunnel equilibrium strongly depends on the interaction between the rock mass and the lining, the convergence-confinement method was found to be a useful tool to illustrate the effects of the main degradation processes on the long-term stability conditions of the tunnel. In that framework, some examples are presented and the results are discussed in terms of safety factor evolution with time.

\section{Long-term analyses}

Due to its simplicity, although limited by basic assumptions (plane strain and axisymmetric conditions), the convergence-confinement method is quite widespread and well developed. Through a graphical representation of the lining pressure $p_{\mathrm{i}}$ versus the radial wall displacement $\delta_{\mathrm{R}}$, it is possible to determine the equilibrium of a tunnel by the intersection of two curves (Fig. 1):

- the convergence line (or ground characteristic curve, GCC), which represents the rock mass response,

- the confinement line (or support reaction line, SRL), which describes the supporting structure reaction.

A detailed literature review on the convergence-confinement method has been given in Brown et al. (1983), Panet (1995), Carranza-Torres and Fairhurst (2000) and Park and Kim (2006).

Since the beginning of the use of the convergenceconfinement method, time was identified as an important factor to be considered for properly evaluating the tunnel equilibrium conditions. Indeed by introducing rock mass and lining degradations in the calculation of the characteristic lines, the tunnel equilibrium (i.e. the intersection point between the GCC and the SRL) evolves with time as shown in Fig. 1.

Although the construction proposed by the convergence-confinement method is widespread, it is essential to keep in mind that it is based on restrictive assumption. A more rigorous mechanical approach to the long-term rock support interaction analysis would require a numerical model (e.g. FEM) for validating that the point of intersection of the convergence line GCC and the confinement line SRL represents indeed the equilibrium point.

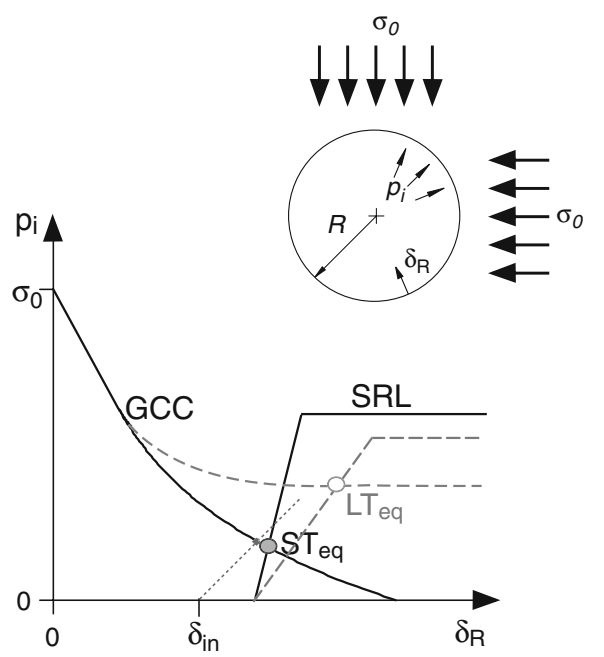

Fig. 1 Ground characteristic curve $(G C C)$ and support reaction line $(S R L)$ in a convergence-confinement graphical representation. The solid lines and their intersection, i.e. the equilibrium point, $S T_{e q}$, are related to short-term conditions. The dashed lines and their intersection, $L T_{e q}$, refer to long-term conditions 
Table 1 Summary of existing solutions for considering the time effects on the rock mass behaviour described by the ground characteristic curve

\begin{tabular}{|c|c|c|}
\hline References & Constitutive model (rock mass) & Special feature (long-term) \\
\hline \multicolumn{3}{|l|}{ Ageing } \\
\hline Panet (1979) & Viscoelastic (Kelvin-Voigt model) & Reduction in Young's Modulus with time \\
\hline Ladanyi (1980) & Elastic-non-linear viscoelastic & $\begin{array}{l}\text { Creep law, distinguishing time and stress effects: primary } \\
\text { creep (both power and logarithmic laws) and secondary } \\
\text { creep (steady-state) }\end{array}$ \\
\hline Berest and Nguyen Minh (1983) & Elastic-viscoplastic (Bingham-Norton model) & $\begin{array}{l}\text { Two solutions for viscoplastic behaviour of the excavated } \\
\text { rock mass, based on the lining stiffness }\end{array}$ \\
\hline Fritz (1984) & $\begin{array}{l}\text { Elastic-viscoplastic (Bingham-Norton model) } \\
\text { with peak and residual conditions in } \\
\text { plasticity (Mohr-Coulomb) }\end{array}$ & $\begin{array}{l}\text { Semi-analytical solution by considering a plastic criterion } \\
\text { (peak and residual conditions) }\end{array}$ \\
\hline $\begin{array}{l}\text { Cristescu (1985), Cristescu (1988), } \\
\text { Cristescu (1994), Cristescu and } \\
\text { Hunsche (1998) }\end{array}$ & $\begin{array}{l}\text { Linear viscoelastic, linear elastic-linear } \\
\text { viscoelastic, linear elastic-non-linear } \\
\text { viscoelastic, elastic-viscoplastic }\end{array}$ & $\begin{array}{l}\text { Constitutive model for a horizontal tunnel, with and } \\
\text { without supporting structure }\end{array}$ \\
\hline Sulem et al. (1987), Sulem (1994) & $\begin{array}{l}\text { Elastic perfectly plastic with softening } \\
\text { (Mohr-Coulomb)/linear viscoelastic } \\
\text { behaviour (Kelvin-Voigt model) }\end{array}$ & $\begin{array}{l}\text { Modelling creep, distinguishing time and face effects time } \\
\text { with logarithmic law }\end{array}$ \\
\hline Barla (2001) & Elastic-plastic with softening & $\begin{array}{l}\text { Modelling rock mass squeezing behaviour by using strain } \\
\text { softening law }\end{array}$ \\
\hline Rousset (1990) & $\begin{array}{l}\text { Elastic-plastic and viscoplastic with } \\
\text { softening (Mohr-Coulomb) }\end{array}$ & $\begin{array}{l}\text { Time-dependent softening after passing the limit of } \\
\text { viscoplastic strain }\end{array}$ \\
\hline Nguyen Minh and Pouya (1992) & $\begin{array}{l}\text { Elastic-viscoplastic (i.e. "non-linear } \\
\text { viscoelastic") }\end{array}$ & $\begin{array}{l}\text { Creep law, by separating time and stress effects: secondary } \\
\text { creep (steady-state) described by Norton's law and } \\
\text { primary creep described by Lemaitre's law (time power } \\
\text { law) }\end{array}$ \\
\hline Bultel (2001) & $\begin{array}{l}\text { Elastic perfectly plastic (Mohr-Coulomb) } \\
\text { without dilatancy }\end{array}$ & Swelling law by (Gysel 1987) \\
\hline Boidy (2002) & $\begin{array}{l}\text { Elastic-viscoplastic (i.e. "non-linear } \\
\text { viscoelastic") }\end{array}$ & $\begin{array}{l}\text { Primary creep, modelled with Lemaitre's law, } \\
\text { distinguishing time and stress effects }\end{array}$ \\
\hline \multicolumn{3}{|l|}{ Weathering } \\
\hline Ladanyi (1974) & Elastic-plastic & Reduction in strength properties with time (isochrones) \\
\hline Daemen (1975) & $\begin{array}{l}\text { Elastic-plastic with softening } \\
\quad \text { (Mohr-Coulomb) }\end{array}$ & Effects of strength reduction on support system loading \\
\hline $\begin{array}{l}\text { C. Carranza-Torres and } \\
\text { M. Diederichs, personal } \\
\text { communication (2005) }\end{array}$ & $\begin{array}{l}\text { Elastic-plastic with softening } \\
\text { (Mohr-Coulomb and Hoek-Brown) }\end{array}$ & Reduction in rock mass strength and stiffness properties \\
\hline \multicolumn{3}{|l|}{ Other actions } \\
\hline Gärber (2003) & $\begin{array}{l}\text { Elastic-plastic (Mohr-Coulomb) } \\
\quad(\text { dilatancy } \psi=0 \text { or } 0.5 \varphi)\end{array}$ & $\begin{array}{l}\text { Redistribution of pore water pressure after tunnel } \\
\text { construction (New Design Method) }\end{array}$ \\
\hline Carranza-Torres and Zhao (2008) & Elastic-plastic (Tresca) & $\begin{array}{l}\text { Mechanical effects of changes in pore pressure due to } \\
\text { drainage }\end{array}$ \\
\hline
\end{tabular}

For each degradation process the list is given in a chronological order

\subsection{Long-term convergence line}

Several authors tried to include time and degradation effects in the basic equations of the characteristic lines. Table 1 summarises contributions related to the evolution of the GCC with time.

\subsubsection{Ageing}

Rock mass ageing has been modelled by several authors in the past by means of different rheological models: Maxwell and Kelvin-Voigt (Panet 1979), Bingham (Berest and
Nguyen Minh 1983), Rousset (Rousset 1990). In 1980, under the hypothesis of a time function independent of stresses, Ladanyi (1980) modelled the creep behaviour of the rock mass by a non-linear viscoelastic model. A similar approach was used by Sulem et al. (1987) for simplifying the convergence measurements interpretation during tunnel excavation. As the tunnel side walls still continued to converge when the face was stopped, they separated rock mass rheological behaviour (i.e. time effects) from the face advancing effects. Thus, according to this approach, when the tunnel excavation is completed, the rheological behaviour of the rock mass may still contribute to changing 
the final equilibrium conditions of the tunnel. By introducing a new general constitutive law, Cristescu (1985, 1994) and Cristescu and Hunsche (1998) developed several parametric analyses for a circular excavation. Both viscoelastic and viscoplastic behaviours of the rock mass were considered.

Without considering the time-dependent behaviour of the tunnel due to face advancing, Berest and Nguyen Minh (1983) analysed the viscoplastic behaviour of the excavated rock mass by considering two different types of lining. As expected, the final convergence of the rock mass is a function of the stiffness of the supporting structure: for a smaller stiffness the rock mass strains increase. Under the condition of non-compressible material, Nguyen Minh and Pouya (1992) proposed an approximated solution for describing the steady-state creep by means of Norton's law. In his Ph.D. thesis, Boidy (2002) used the same solution for evaluating viscous strains in the excavated rock mass induced by primary creep as described by Lemaitre's law. As far as viscoplastic models are concerned, further considerations are required. Due to its formulation, the viscoplastic model, as proposed by Nguyen Minh and Pouya (1992) and Boidy (2002), does not take into consideration the rock mass failure criterion. In fact, it rather corresponds to a non-linear viscoelastic model according to the definition of Ladanyi (1980). As a matter of fact, a complete formulation for describing elastic-viscoplastic behaviour requires strength parameters, as introduced by Rousset (1990). Fritz (1984) developed a complete semi-analytical solution for a circular tunnel excavated in an elastoviscoplastic rock mass with a Mohr-Coulomb criterion. The viscoplastic strains develop only in the plastic zone around the excavation.

As part of the tunnel ageing pathologies, the squeezing behaviour of the rock mass has also been considered. Barla (2001) suggested introducing strain softening laws in the convergence-confinement analyses for modelling the longterm behaviour of tunnels in squeezing rock masses. According to this approach, in the long-term, the plastic zone around the tunnel is characterised by residual strength parameters. Finally, swelling behaviour was modelled by Bultel (2001). According to the simplified approach proposed by Gysel (1987), the swelling behaviour of a rock mass induces volumetric strain proportional to the ratio of the mean stress and the swelling limit.

\subsubsection{Weathering}

Based on uniaxial compression tests performed by several authors, Ladanyi (1974) stated that the rock mass mechanical characteristics may decrease in the long term due to weathering. By solving the GCC equations using long-term parameters, he drew several isochrones corresponding to a given level of decrease in properties and, thus, to a certain time after tunnel excavation. Unfortunately, though clearly identified, the time dependency of the decrease in mechanical parameters is not properly described by any equation and may change from tunnel to tunnel. Daemen (1975), in the framework of the convergence-confinement method, developed a numerical solution for evaluating the change in support loading conditions due to reduction in rock mass strength properties with time caused by weathering agents. Assuming a decrease in strength and stiffness properties of the rock mass due to weathering that is proportional to the plastic volumetric strain around the tunnel, C. Carranza-Torres and M. Diederichs (2005; personal communication) proposed solutions for an elastic-plastic rock mass with softening behaviour, considering both Mohr-Coulomb and Hoek-Brown criteria.

\subsubsection{Other actions}

Pore water pressure redistribution with time is one of the other mechanical actions that affect tunnels driven in water-saturated rock masses. By considering this redistribution of pore water pressure in low permeable saturated porous media, Gärber (2003) in his Ph.D. thesis proposed a new design method for evaluating the long-term pressure on the tunnel lining by considering the water boundary conditions at the gallery wall (permeable or impermeable lining). Recently, Carranza-Torres and Zhao (2008) developed an analytical solution that considers the longterm mechanical effects of pore pressure changes on the mechanical response of a tunnel. Both permeable and impermeable linings are considered. The proposed solution is uncoupled and considers only the post-transient conditions.

\subsection{Long-term confinement line}

Probably due to the fact that the convergence-confinement method is often used for interpreting convergence measurements during tunnel construction, i.e. when delayed phenomena mainly depend on the rock mass rheological behaviour, very few attempts have been made in the past to introduce the time effects on the confinement line. Oreste and Peila (1997) and Oreste (2003) proposed a method for evaluating transient conditions during hardening of shotcrete support and representing this time evolution using confinement lines. The values of shotcrete elastic modulus and compressive strength are progressively increased by means of an iterative process that covers the construction period. 


\section{Convergence-confinement solutions considering tunnel degradation processes}

\subsection{Progressive load transfer from the primary support} to the final lining

As tunnel conditions evolve with time, safety and serviceability must be checked during the entire service life of the tunnel: i.e. during construction (short term) and during operation (medium to long term). In particular, three steps can be defined for characterising the tunnel life:

- short term, which represents the period during and after construction when the primary support is active;

- medium term, some years after construction, when the primary support is fully degraded and the rock load is supported only by the final lining;

- long term, during which the final lining degrades until the end of the service life of the tunnel (about 90 years after its construction, SIA 197 2004).

Since in the convergence-confinement method, the tunnel equilibrium is represented by the intersection between the ground convergence curve and the confinement line, some considerations are required to explain the changing conditions of the primary support/final lining interaction with the excavated rock mass. Primary support is applied immediately after the tunnel excavation to ensure safe working conditions and to mobilise the rock mass strength by controlling displacements. The construction is then completed with a final lining. Assuming, as it is commonly done in the literature (Panet 1995), that if several supports are installed at the same time the total stiffness of the system is represented by the sum of the stiffness of each support; similar considerations may be applied when the final lining is completed. Obviously as a time delay occurs between the installation of the systems, the construction of the SRL should take into consideration the displacements that occurred during this time interval. Thus, the new equilibrium depends on the total stiffness, on the displacement that occurred before placing the primary support and on the displacement of the primary support at the moment of the final lining installation. When a waterproofing membrane is placed between the primary support and the final lining, the primary support degrades much faster than the final lining does, due to the corrosive action of groundwater and soil. Primary support contribution to equilibrium conditions is neglected in the medium and long terms. Figure 2 shows the main steps that describe the progressive transfer of the rock mass load from the primary support to the final lining system:

1. At the beginning, the equilibrium depends only on the primary support characteristics $\left(k_{\mathrm{s}}\right)$.
2. When the final lining is completed, both the primary and the final supports act together, with a progressive degradation of the primary support $\left.\left[k_{\mathrm{s}}(t)+k_{\mathrm{i}}\right)\right]$. The final lining is affected by a much slower degradation, which can be neglected during this phase.

3. In the medium term, the primary support is fully degraded and only the final lining contributes to tunnel stability $\left(k_{\mathrm{i}}\right)$.

In the long term, the final lining is also affected by degradation $\left[k_{\mathrm{i}}(t)\right]$. To evaluate the effects of long-term degradation on the evolution of tunnel conditions, it is necessary to take into consideration:

- all the ageing factors, which can be modelled by stiffness reduction or strain increase;

- all the aggressive agents, including water action, which mainly contribute to tunnel weathering and which can be modelled by strength and/or, in the case of final tunnel lining, by thickness reduction;

- all other mechanical actions that may change the stress conditions (e.g. pore water pressure redistribution and consolidation).

According to the time-dependent representation introduced by Ladanyi (1974), and considering that 90 years correspond to the end of the service life of the tunnel, as suggested by the Swiss norm (SIA 197 2004), changing conditions from short to long term are represented by means of isochrones. With this kind of representation, the curves describe the characteristic lines in the short, medium and long terms: i.e. at tunnel construction, when the rock mass load transfer from the primary support to the final lining is completed, and 10, 30 and 90 years after construction.

The results (i.e. changing equilibrium conditions) have been interpreted in terms of a global safety factor defined as the ratio between the maximum pressure that the supporting structure can bear, $p_{\max }$ and the actual equilibrium pressure, $p_{\text {eq }}$ :

$\mathrm{FoS}=\frac{p_{\mathrm{max}}}{p_{\mathrm{eq}}} \geq 1$

As observed by Panet (1995), the support behaviour changes depending on the type of support. For example, a primary support composed of steel sets can be easily associated to an elastic, perfectly plastic behaviour characterised by a considerable ductile strain when compared to a shotcrete ring (without fibres). The maximum load that the supporting structure can bear is evaluated by considering the material strength. The contribution of each element is considered also when it is yielded until its final failure. Thus, with the same simplifying assumption done for the stiffness, the maximum pressure $p_{\max }$ depends on 
Fig. 2 Progressive transfer of the rock mass load from the temporary support to the final lining system due to support degradation. Evolution of the tunnel equilibrium from the short to the medium-term conditions. For clarity, the evolution of the ground characteristic curve with time is not drawn in the figure
1
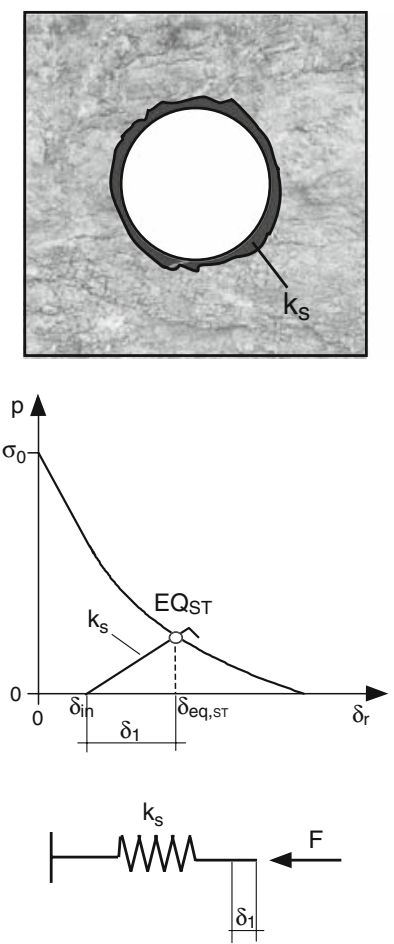

$\mathrm{F}=\mathrm{k}_{\mathrm{S}} \cdot \delta_{1}$

$\delta_{1}=\delta_{\text {eq,ST }}-\delta_{\text {in }}$ the sum of the final strength of all elements that contribute to tunnel stability and on their deterioration with time. As explained earlier, while the short term depends only on the primary support, between the short and the medium term primary support and final lining contribute together to the tunnel stability. Then, when the transfer of the rock mass load from the primary support to the final lining is completed (i.e. the temporary support is completely degraded, medium term), the tunnel stability is assured only by the final lining, which also degrades in the long term. Thus, the tunnel safety factor not only depends on the evolution with time of the equilibrium pressure $p_{\text {eq }}$, but also on the variation in maximum pressure $p_{\max }$ related to the progressive degradation of the elements that contribute to the stability.

\subsection{Rock mass degradation}

\subsubsection{Ageing}

Convergences of side walls and time-dependent displacements of the rock mass after tunnel excavation are generally associated with creep and usually modelled by strain increase (Constantinescu and Cristescu 1983). This strain depends mainly on the deviatoric stress; deformation velocity and type are also strongly correlated to this stress.
2
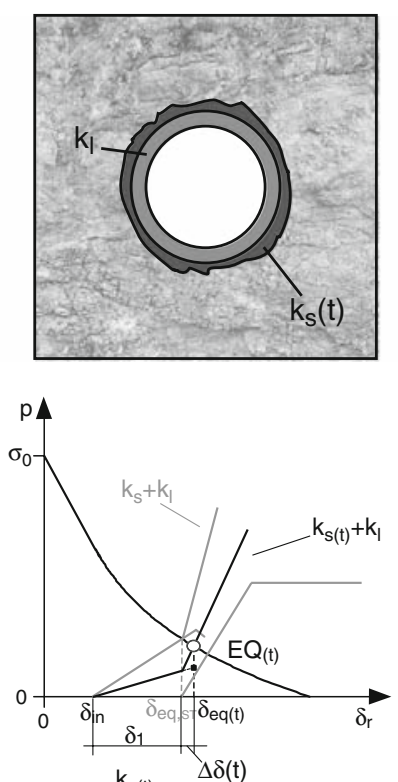

$\mathrm{k}_{\mathrm{s}(\mathrm{t})} \quad \Delta \delta(\mathrm{t})$

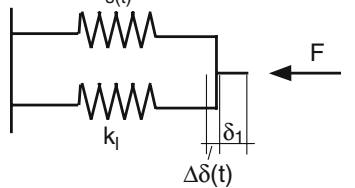

$\mathrm{F}=\mathrm{k}_{\mathrm{S}}(\mathrm{t}) \cdot\left(\delta_{1}+\Delta \delta(\mathrm{t})\right)+\mathrm{k}_{\mathrm{l}} \cdot \Delta \delta(\mathrm{t})$

$F=k_{s}(t) \cdot \delta_{1}+\left(k_{s}(t)+k_{1}\right) \cdot \Delta \delta(t)$
3
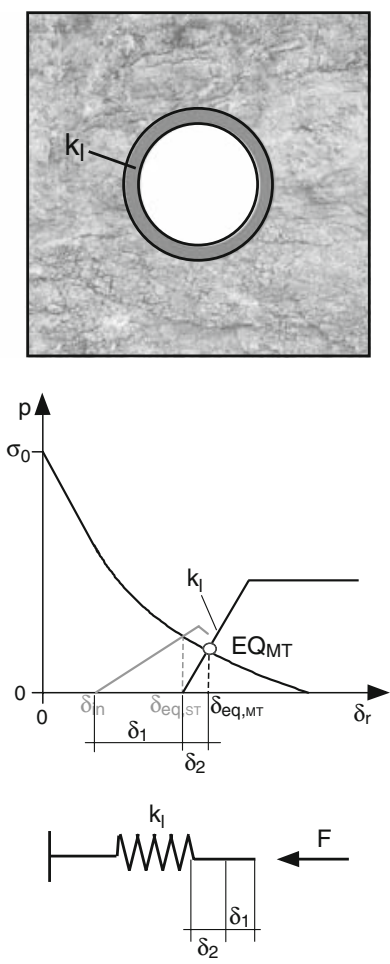

$\mathrm{F}=\mathrm{k}_{\mathrm{l}} \cdot \delta_{2}$

$\delta_{2}=\delta_{\text {eq,MT }}-\delta_{\text {eq,ST }}$
Ageing speed can be very different from one kind of rock to another. Among several of the models tried in the past and reported in Table 1, Lemaitre's law will be considered here. This law models the primary creep by means of a viscoplastic strain, $\varepsilon_{\mathrm{vp}}$ function of the deviatoric part of the stress tensor (Boidy 2002; Boidy et al. 2002):

$\varepsilon_{\mathrm{vp}}=a \cdot\left(q-\sigma_{\mathrm{s}}\right)^{\beta} \cdot t^{\alpha}$

where

- $q$ is the deviatoric stress in the rock mass, expressed in $\mathrm{MPa}$;

- $\sigma_{\mathrm{s}}$ is a limit stress beyond which the delayed behaviour starts. It expresses the plastic criterion and is not taken into consideration in this case. Actually, by considering $\sigma_{\mathrm{s}}=0 \mathrm{MPa}$, the model has non-linear viscoelastic behaviour as defined by Ladanyi (1980). For the sake of simplicity, in the following it will be referred to as a viscoplastic model;

- $t$ is the time, expressed in $\mathrm{s}$;

- $\alpha$ is a creep constant between 0.3 and 1 (note that $\alpha=1$ is the Norton's law describing secondary creep) as quoted by Ladanyi (1980);

- $\quad \beta$ is a constant depending on the deformation mechanism and, thus, on rock mass and creep type; 
- $a=\left(\frac{A}{\alpha}\right)^{\alpha}$, with $A$, expressed in $\mathrm{s}^{-1}$ the viscosity parameter, usually described by Arrhenius' law.

Using the approximate solution proposed by Nguyen Minh and Pouya (1992) and Boidy (2002), the long-term ground reaction curves can be drawn as follows:

- the stress evaluated by integrating the equation, which describes the equilibrium of a cylindrical cavity under axisymmetric load and plane strain conditions;

- the displacement evaluated by integrating the deformation around the excavation.

\subsubsection{Weathering}

According to Brady and Brown (2004), the interaction between rock mass exposed surfaces and groundwater is similar to weathering of conventional building materials (e.g. concrete). During the past few years, as reported in Hagros et al. (2007), several authors showed that the chemical interaction between rock and groundwater reduces the rock strength properties. Compared to other geological formations, sedimentary rocks are particularly sensitive to water action (both physical and chemical) and to oxidation. This is due mainly to their porosity, which allows air and water to flow through them. Based on uniaxial compression tests performed by several authors, Ladanyi (1974) stated that due to chemical weathering, the strength properties of a rock mass may be reduced in the long term by about $30 \%$ of their initial value. Today, this reduction is commonly used in Rock Mechanics for evaluating long-term strength parameters. In the present paper, rock mass weathering is modelled by reducing strength properties with time according to a hyperbolic law, as commonly used for describing time influence on ground properties (Ladanyi 1974; Sulem 1994):

$V(t)=V_{\mathrm{ST}}-\left[\left(V_{\mathrm{ST}}-V_{\mathrm{LT}}\right) \cdot\left(1-\frac{1}{1+\frac{t}{T}}\right)\right]$

where $V_{\mathrm{ST}}$ is the short-term value of the parameter (i.e. friction angle $\varphi$ and cohesion $c$ ), $V_{\mathrm{LT}}$ is the value in the long term of the same parameter, considering a reduction by $30 \%$ of its initial value $\left(V_{\mathrm{ST}}\right), t$ is the time elapsed since tunnel construction, and $T$ is a constant that defines the rate of the rock mass weathering process.

The influence of rock mass deterioration on the long-term loading conditions of tunnel crowns has been discussed by Caquot and Kerisel (1956) for shallow tunnels. The decrease in quality and mechanical properties of the rock mass results in an additional load on the lining due to gravity. This may cause lining cracking and, in worse cases, the stability conditions of the tunnel can be reduced as to lead to lining failure and tunnel collapse, as described in (Széchy 1966; CETu
2004). Terzaghi (1946) defined the rock load as the height of the mass of rock which tends to drop out of the roof (i.e. the damaged zone around an excavated tunnel). Moreover, he estimated how the size and the shape of this zone depend on the geotechnical conditions of the rock mass. In the convergence-confinement method (Panet 1995), the broken zone is quite often associated to the damaged (yielded) zone around the tunnel. As far as short-term analyses are concerned, Pacher (1964) suggested a conservative correction of the load on the tunnel crown by simply considering the weight of the plastic zone:

$\Delta p_{\text {crown }}=\gamma_{\mathrm{rm}} \cdot\left(R_{\mathrm{pl}}-R\right)$

with

- $\gamma_{\mathrm{rm}}$ unit weight of the rock mass above the tunnel crown,

- $R$ tunnel radius and $R_{\mathrm{pl}}$ plastic radius.

In the long term, due to weathering processes, the weathered zone above the tunnel crown grows and so does the load that the lining has to bear. The long-term crown convergence curve can be drawn as suggested by Pacher (1964) with an additional pressure equal to the weight of the broken zone.

\subsection{Concrete lining degradation}

Since all Swiss National Road tunnels, due to their age, have concrete or reinforced concrete lining, in this framework only the pathologies affecting concrete will be considered.

Like other materials, concrete is affected by long-term degradation: it usually begins on exposed surfaces, and, under water pressure, and may accelerate through cracks (Grobbelaar 1994). Thus, although at the beginning of the tunnel service life, concrete mechanical properties improve, degradation rate is predominant in the long term. Several weathering factors contribute to modifying the final lining characteristics during tunnel operation. The degradation rate of the final lining may change a lot from tunnel to tunnel, mainly due to the position of the waterproofing system. Two different models of concrete lining weathering have been considered:

1. When the waterproofing system is absent or does not work properly, the final lining is exposed to external attacks at both the intrados and the extrados. This process causes reduction in mechanical properties due to possible leaching of aggressive groundwater from the extrados to the intrados of the lining (e.g. sulphates corrosion, Calcium leaching...).

2. When a waterproofing membrane is placed at the final lining extrados, under normal condition, the only 
active surface in terms of degradation is the lining intrados. This process causes reduction in the final lining thickness, which is mainly caused by de-icing salts corrosion.

Though the symptoms of these degradation processes are mainly locally weathered surfaces, from a general point of view it is possible to affirm that both processes may result in changing the long-term behaviour of the final lining structure.

\subsubsection{Calcium leaching}

An example of concrete weathering due to Calcium leaching is shown in Fig. 3. The dissolution of soluble cement constituents due to water leakage is described by reactions (Nguyen 2005) where carbon dioxide, emanating from exhaust gases from traffic, can be in a free form (i.e. $\mathrm{CO}_{2}$ ) or linked with water (i.e. $\mathrm{HCO}_{3}$ ):

$$
\begin{aligned}
& \mathrm{Ca}(\mathrm{OH})_{2}+\mathrm{CO}_{2} \leftrightarrow \mathrm{CaCO}_{3}+\mathrm{H}_{2} \mathrm{O} \\
& \mathrm{CaCO}_{3}+\mathrm{CO}_{2}+\mathrm{H}_{2} \mathrm{O} \leftrightarrow \mathrm{Ca}\left(\mathrm{HCO}_{3}\right)_{2} .
\end{aligned}
$$

Calcium leaching increases the porosity and, consequently, the permeability of the concrete lining. This process reduces both the stiffness and the strength of the concrete lining. Moreover, the micro-fissuration may evolve into the formation of cracks (see Fig. 3 right), accelerating the exchanges between the tunnel and the external environment and increasing the degradation speed of the concrete lining. Carde and Francois (1997) and Nguyen (2005) showed through laboratory tests that the decrease in mechanical properties is directly proportional to the ratio between the degraded area $A_{\mathrm{d}}$ and the original section size $A_{0}$ :

$\delta A_{\mathrm{d}}=\frac{A_{\mathrm{d}}}{A_{0}}$

According to Nguyen (2005), the evolution of the Young's Modulus $E$ and the compressive strength $f_{\mathrm{c}}$ of the concrete lining can be described as follows: $\frac{\delta E}{E_{0}}=\frac{E_{0}-E}{E_{0}}=k_{\mathrm{m}} \times \delta A_{\mathrm{d}}$

$\frac{\delta f_{\mathrm{c}}}{f_{\mathrm{c}_{0}}}=\frac{f_{\mathrm{c}_{0}}-f_{\mathrm{c}}}{f_{\mathrm{c}_{0}}}=k_{\mathrm{r}} \times \delta A_{\mathrm{d}}$

where $E_{0}$ and $f_{\mathrm{c}_{0}}$ represent the Young's Modulus and the compressive strength of the original material, while $k_{\mathrm{m}}$ and $k_{\mathrm{r}}$ are, respectively, 0.66 and 0.76 for a mediumquality concrete. Usually, the thickness of the degraded zone, $X_{\mathrm{d}}$, is considered proportional to the square root of time, $t$ :

$X_{\mathrm{d}}=a(\sqrt{t})$.

The degradation speed $a$ is a material constant that depends on the water to cement ratio (W/C). Yokozeki et al. (2004) analysed the behaviour of structures aged between 34 and 104 years, and stated that the degradation of the thickness due to Calcium leaching can reach a maximum value of $100 \mathrm{~mm}$ in 100 years. A similar rate has been obtained by analysing the results of laboratory tests conducted on specimens bored in the concrete lining of the Flonzaley tunnel (Vaud Canton, Switzerland) after about 30 years of service life. The uniaxial compressive tests performed on these samples give a mean compressive strength of the concrete $f_{\mathrm{c}}$ of about $39 \mathrm{MPa}$, to be compared to an initial strength value $f_{\mathrm{c}_{0}}$ of $45 \mathrm{MPa}$. Thus, considering that the degraded thickness, $X_{\mathrm{d}}(\mathrm{m})$, is proportional to the square root of time, $t$ (days), as expressed in Eq. 10, it is possible to estimate the degradation rate for a concrete with a water to cement ratio equal to 0.5 . As a result, $a \cong 5.2 \mathrm{E}-4$ $\left(\frac{m}{\sqrt{\text { days }}}\right)$ (Fig. 4). For a tunnel without a waterproofing system or with a defective membrane, weathering of the final lining caused by aggressive groundwater can be modelled, by reducing the mechanical properties of the concrete as expressed by Eqs. 8 and 9, considering that the degraded area, $A_{\mathrm{d}}$, corresponds to a degraded thickness, $X_{\mathrm{d}}$, per meter of length.
Fig. 3 Calcium leaching (courtesy of Vaud Canton, Switzerland). On the right, an enlargement
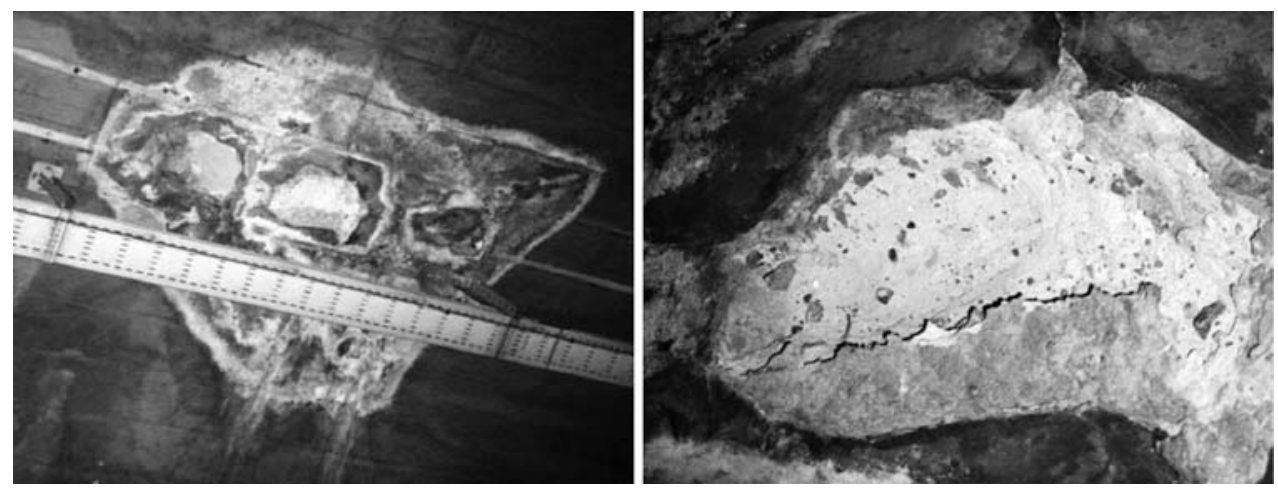


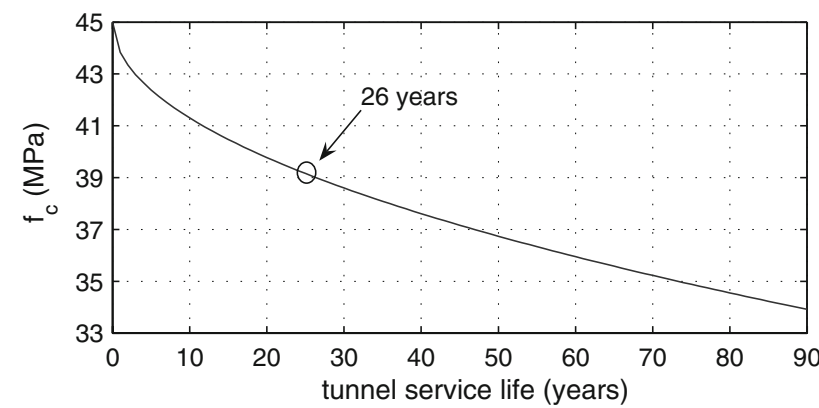

Fig. 4 Decrease of compressive strength $f_{\text {c }}$ with time due to aggressive groundwater leaching through the tunnel lining. The curve describes the weathering process that affects the concrete lining of the Flonzaley tunnel (Vaud Canton, Switzerland) caused by water incomes due to a defective waterproofing membrane

\subsubsection{De-icing salt corrosion}

When the final lining extrados is protected from the weathering agents coming from the rock mass by a waterproofing system, the lining intrados is the only active surface for chemical degradation. The use of de-icing salts (i.e. mainly Chlorides solutions, $\mathrm{CaCl}_{2}$ and $\mathrm{NaCl}$ ) may have different consequences (Kaufmann 2000; Wang et al. 2006):

- concrete saturation and risk of frost damage increase;

- decrease in the freezing point of the pore solution;

- chemical deterioration due to interaction between de-icing chemicals and concrete material.

As in any other concrete structure, in a plain concrete lining it is possible to distinguish the surface ("skin") characterised by a lower concrete quality, and the core of the structure with a higher concrete quality. Being influenced by the concrete quality, the skin degrades faster than the core. After several applications of de-icing salts, the maximum content of Chlorides is found at a certain depth from the concrete surface (e.g. about $40-50 \mathrm{~mm}$ ). Due to the lowering of freezing point by the salt solution, the concrete structure can be divided into different layers, which would freeze at different temperatures. This may induce unexpected tensile stresses in the structure and cause concrete surface scaling. In particular, during cold winters and with frequent use of de-icing salts, this process can repeat itself and the degradation of the concrete structures is accelerated, reducing the final lining thickness. Moreover, chemical deterioration accelerates with increase in permeability due to micro- and macro-cracking of concrete.

Based on prescriptions for concrete structures (SN EN 206-1 2000), it is possible to identify for each part of the concrete lining the exposure class to de-icing salts. Depending on the effects of rolling traffic, two different

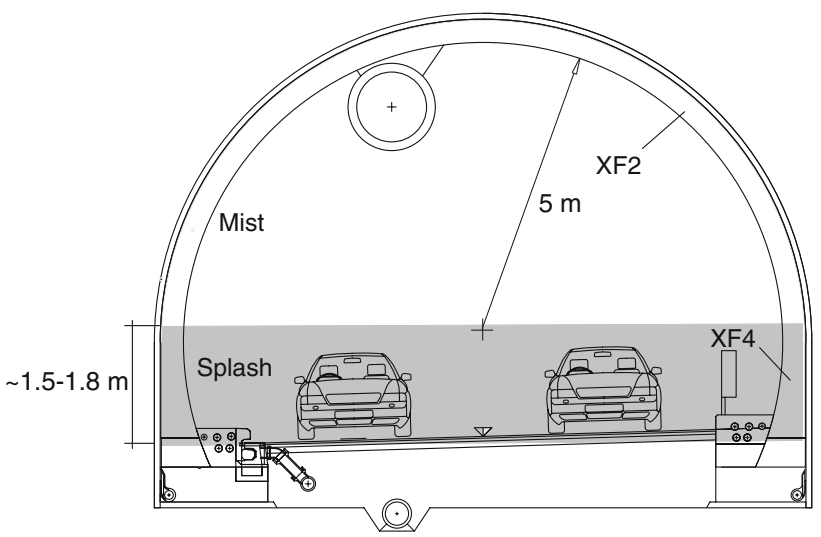

Fig. 5 Exposure of a plain concrete tunnel lining to de-icing salts. For a typical highway tunnel section, according to the exposure classes proposed for bridges in SN EN 206-1 (2000), rolling traffic may project Chlorides up to $1.5-1.8 \mathrm{~m}$ height

exposure zones should be distinguished for a plain concrete lining (Fig. 5):

- XF2-mist: tunnel crown (or if ventilation slab) slightly saturated with de-icing salts;

- XF4-splash: side walls up to $1.5-1.8 \mathrm{~m}$ height strongly saturated with de-icing salts.

The thickness of the degraded area depends on the exposure class and on the regularity of the maintenance. One would expect that only the skin of the final lining (poorer quality) would be affected by scaling due to deicing salts frost attacks during the tunnel service life. The process initiation and effects are influenced by humidity, temperature and salt concentration. In particular, according to Kaufmann (2000), worse conditions are represented by a $\mathrm{NaCl}$ concentration of about 2-4\% with homogeneously saturated porous material that corresponds to the gutter and the lower part of the side walls. Unfortunately, there is still not enough information about the rate of this weathering process in tunnels and only some approximation can be done. Considering a weathered thickness proportional to the square root of time (Eq. 10) the rate of the process has been assessed for the two exposure classes according to observations made in principal inspections of tunnels from the Cantons of Vaud and Ticino. In particular, after 30 years of operation, the side wall thickness is locally reduced by about $50 \mathrm{~mm}$ in the lower part, while the upper part and the crown are less affected, i.e. with a thickness reduction of about $30 \mathrm{~mm}$.

\section{Application examples}

Table 2 summarises the examples of typical rock mass and pathologies of concrete lining degradation considered in 
Table 2 Summary of the examples considered in this section to illustrate typical road tunnels pathologies

$$
\text { Case } 1
$$

Case 2

\begin{tabular}{|c|c|c|}
\hline \multicolumn{3}{|l|}{ Rock mass } \\
\hline Type & Marls & Sandstones \\
\hline Pathology & Ageing (creep) & Weathering \\
\hline Model & Viscoplastic behaviour (strain increase) & $\begin{array}{l}\text { Decrease in mechanical properties decrease } \\
\text { Increase of load on the tunnel crown }\end{array}$ \\
\hline Results & Fig. 6 & Fig. 7 \\
\hline \multicolumn{3}{|c|}{ Plain concrete lining } \\
\hline $\begin{array}{l}\text { Waterproofing } \\
\text { system }\end{array}$ & At the lining extrados & No waterproofing \\
\hline Pathology & De-icing salts attack & Calcium leaching \\
\hline Model & Thickness decrease & Decrease in mechanical properties \\
\hline Results & Fig. 9 & Fig. 8 \\
\hline \multicolumn{3}{|l|}{ Combined } \\
\hline Results & Fig. 10 & Fig. 11 \\
\hline
\end{tabular}

this section. After studying separately rock mass and concrete lining degradation effects, (Sects. 4.1, 4.2; Figs. 6, 7, 8, 9), two examples of combined analysis are presented (Sect. 4.3; Figs. 10, 11).

For an easier comparison of the results of the following analyses, only one set of parameters has been considered for the tunnel:

- tunnel radius, $R=5 \mathrm{~m}$ (circular section);

- tunnel depth, $\mathrm{H}=250 \mathrm{~m}$ (i.e. $H \gg R$, to fulfil the assumption of hydrostatic stress field);

- primary support installed at $D_{0}=2 \mathrm{~m}$ from the cutting face: $0.25 \mathrm{~m}$ thick shotcrete with $E_{\mathrm{s}}=23 \mathrm{GPa}$, $v_{\mathrm{s}}=0.2, f_{c_{s}}=14 \mathrm{MPa}$ (as the primary support should guarantee the tunnel stability during construction, in the very short term, the mechanical characteristics are lower than the respective values for shotcrete after 28 days). The origin of the confinement line for the primary support has been evaluated by using the similarity principle introduced by Corbetta (1990);

- final lining: $0.3 \mathrm{~m}$ thick concrete ring with $E_{\mathrm{c}}=35 \mathrm{GPa}, v_{\mathrm{s}}=0.2, f_{\mathrm{c}}=40 \mathrm{MPa}$. According to the Model Code 1990 (CEB 1993), the lining behaves as an elastic perfectly plastic material (ductile strain $\varepsilon_{\mathrm{u}}=0.4 \%$ for a normal concrete with a compressive strength of about 30-40 MPa);

- without further specification, a waterproofing system is placed at the final lining extrados (i.e. between the primary and the final supports).

Two typical Swiss rock formations have been considered: poor-quality sandstones and good-quality marls (Table 3). The values of cohesion $c$ and friction angle $\varphi$ are representative of the rock mass strength in the range of stresses around the excavation. With a Hoek-Brown criterion, the method is similar but characterised by more
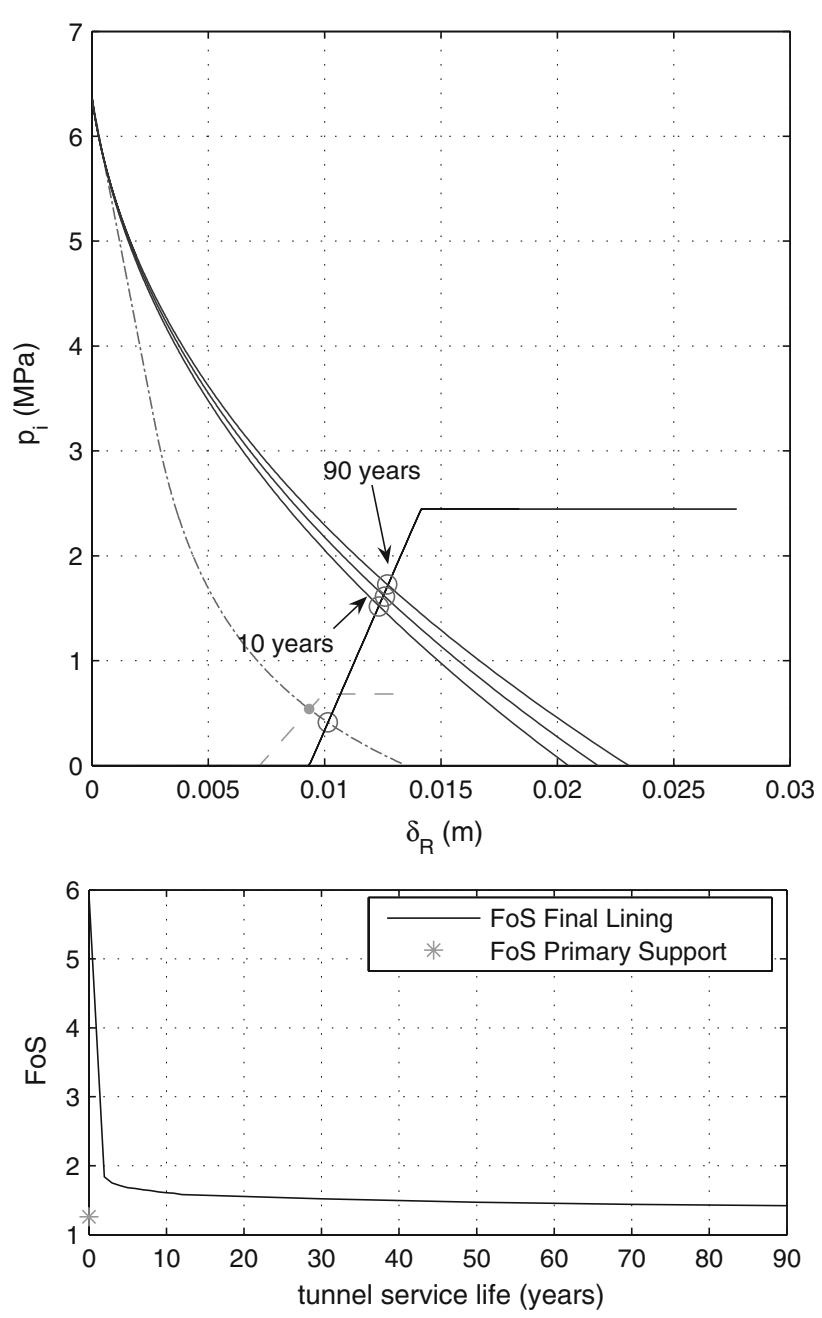

Fig. 6 Top Convergence-confinement analysis with an elasticviscoplastic rock mass (good quality marls, see Table 3 ). Parameters after Boidy et al. (2002). The GCC is represented in the short term and during the service life of the tunnel, respectively, 10, 30 and 90 years after tunnel construction. Bottom FoS evolution with time 

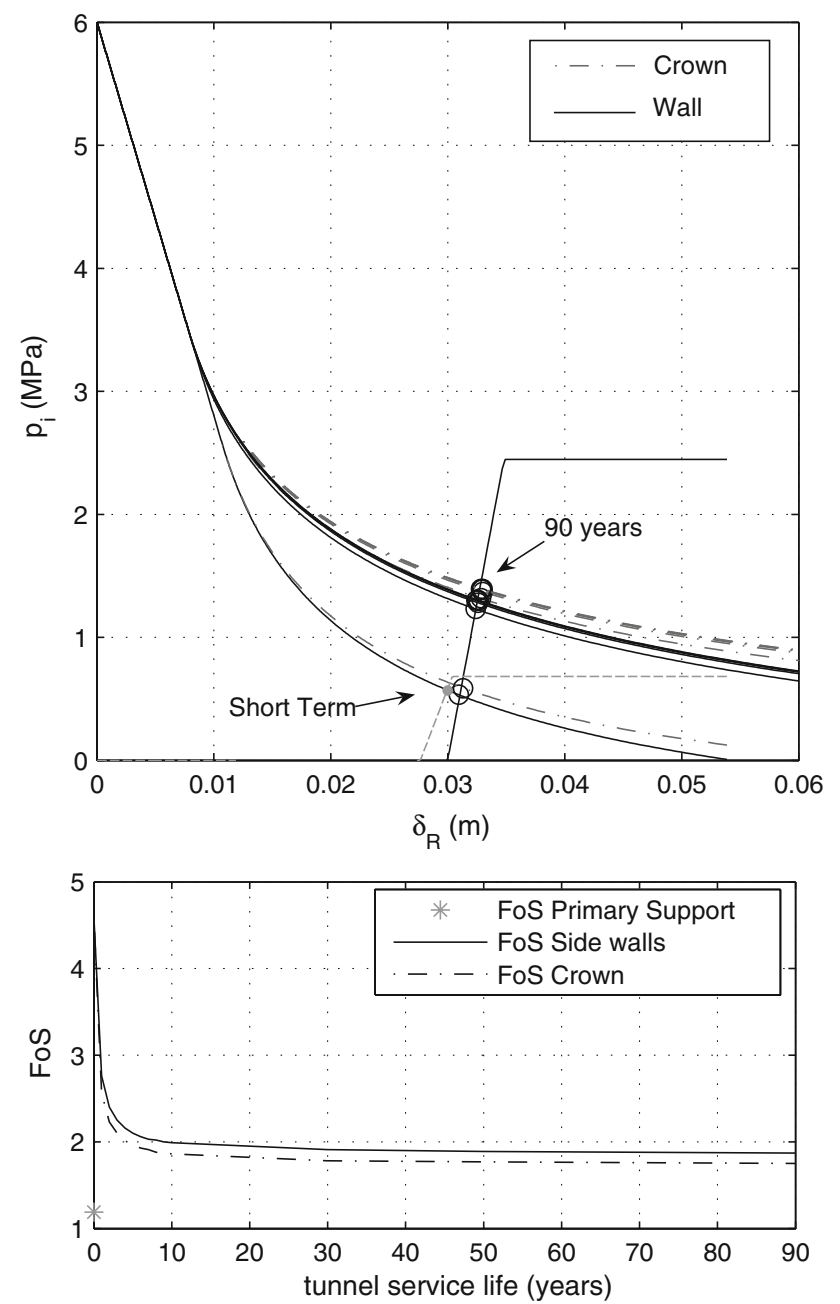

Fig. 7 Top Convergence-confinement analysis of a tunnel excavated in poor quality sandstones (see Table 3), considering weathering effects in the long term (reduction of about $30 \%$ of the short-term strength properties according to the reduction law expressed by Eq. 3 and the roof effects due to the loss of confinement of the excavated rock above the tunnel crown (under the gravity effect). The convergence curves are represented for both side wall (i.e. solid lines) and crown (i.e. dashed lines). Bottom FoS evolution with time

complicated analytical (and numerical) solutions for drawing the GCC.

\subsection{Rock mass degradation}

\subsubsection{Ageing}

Top of Fig. 6 shows in a convergence-confinement diagram the evolution with time of the convergence curve for a $10 \mathrm{~m}$ diameter tunnel excavated at $250 \mathrm{~m}$ depth in good quality marls (see Table 3). The curves were drawn using the approximate solution proposed by Nguyen Minh and Pouya (1992) and Boidy (2002) with the following viscoplastic
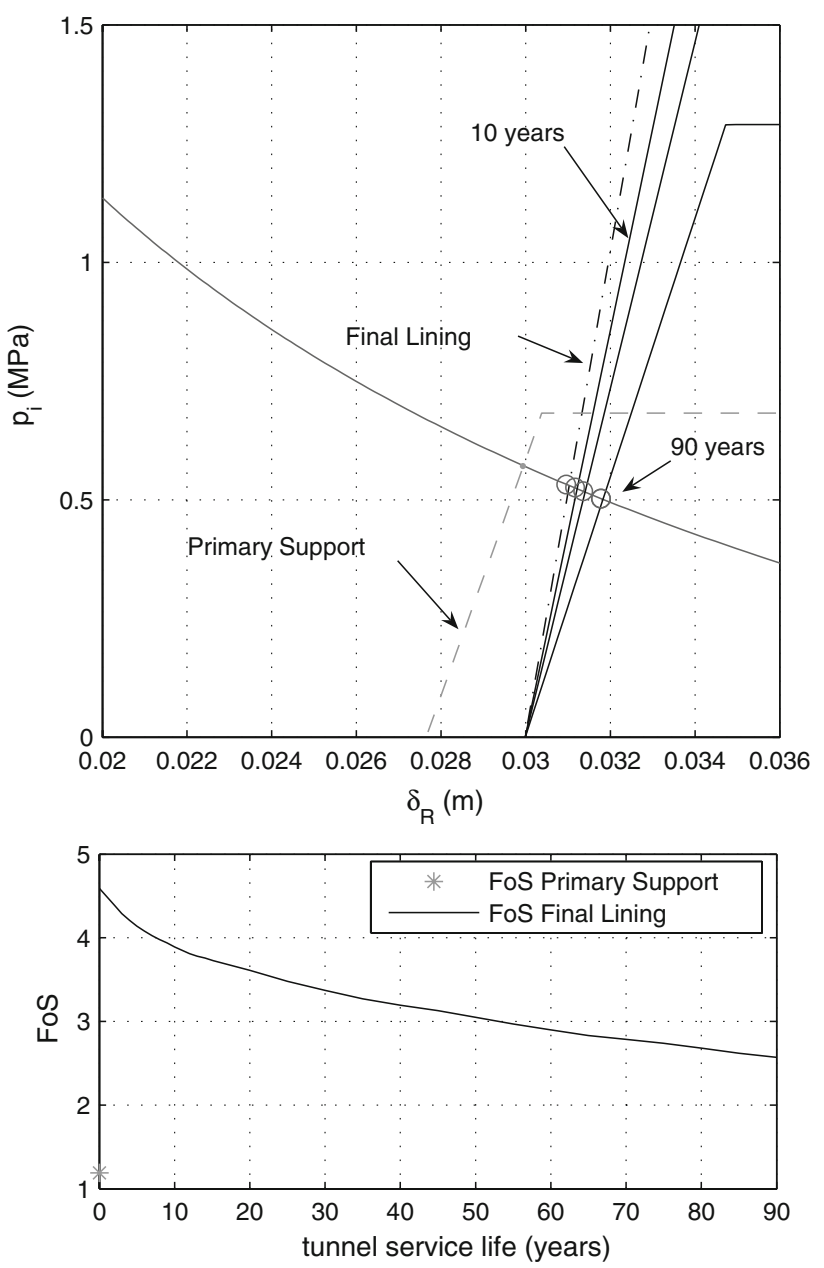

Fig. 8 Convergence-confinement analysis for a tunnel without a waterproofing system excavated in poor quality sandstones (see Table 3). External attack of the concrete lining due to aggressive groundwater leaching. Top Evolution of the lining reaction line. Bottom FoS evolution with time

parameters: $A=3 \mathrm{E}-61, \alpha=0.07$ and $\beta=1.1$. These values were estimated by Boidy et al. (2002) by means of a curve fitting of in situ convergence measurements in the Aalenian marls of the Mont Terri tunnel (Jura Canton, Switzerland).

Bottom of Fig. 6 represents the evolution of the factor of safety (FoS) during the service life of the tunnel. While the safety factor of the temporary support is slightly higher than 1 , when the final lining is laid the initial value of the tunnel safety factor is particularly high (i.e. about 6). This result should be interpreted with particular caution, by noting that the basic assumption that assimilates the lining behaviour to a perfect circular concrete ring, under axisymmetric load, is rarely met in real cases. Nevertheless, a significant decrease of the safety factor with time can be observed. 
Fig. 9 Convergenceconfinement analysis for a tunnel with a waterproofing system excavated in good quality marls (see Table 3 ). Frost de-icing salts attack at tunnel intrados. Top Evolution of the final lining reaction line due to splash exposure (XF4) on the left, and mist exposure (XF2) on the right. Bottom FoS evolution with time
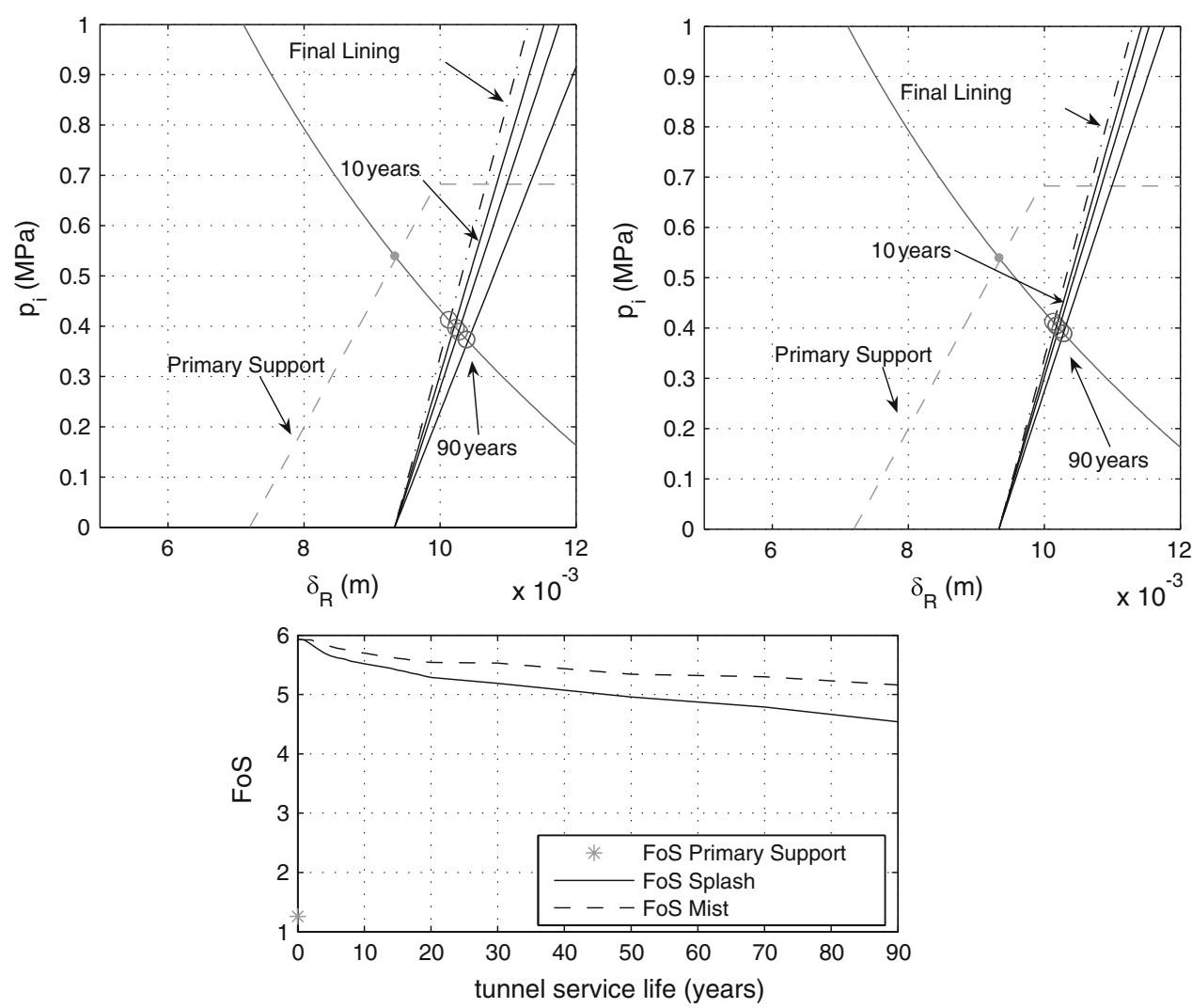

\subsubsection{Weathering}

The weathering of the fissured sandstone massif described in Table 3 has been modelled by reducing its strength properties with time according to Eq. 3, considering $T$, i.e. the constant that defines the rate of the rock mass weathering process, as equal to 1 year, and considering the longterm values of the strength parameters to be equal to $70 \%$ of their short-term values. Figure 7 shows the influence of weathering on the long-term equilibrium of the tunnel.

In the convergence-confinement diagram represented in top of Fig. 7, due to the additional loading of the degraded rock mass above the tunnel roof (Eq. 4), the GCCs describing the behaviour of the tunnel crown (i.e. dashed curves) do not coincide anymore with the GCCs describing the behaviour of the side walls (i.e. solid curves). Thus, in this case, as the pressure distribution around the cavity is not axisymmetric, before estimating the tunnel safety factor, it would be necessary to evaluate the stresses from the thrust and the bending moment in the final lining. Actually, the convergence curves plotted in Fig. 7, show that the difference between the equilibrium in crown and side walls is negligible. Thus, the safety factor (Fig. 7, bottom) was evaluated for crown and side walls separately under the hypothesis of axisymmetric loads. A reduction in the strength properties of about $30 \%$ of their short-term values corresponds to a decrease in the safety factor of more than
$55 \%$. Note that, in this case also, the final lining of the tunnel is not affected by long-term degradation.

It is worthwhile to mention that the above-presented example only considers the reduction in the strength properties (i.e. $c$ and $\varphi$ ) due to chemical weathering, while the Young's Modulus is kept constant. In reality, rock mass weathering may cause a decrease in all the mechanical properties of the weathered zone (i.e. plastic zone). For example, Ladanyi (1974) suggested a reduction in not only the strength properties, but also in the Young's Modulus in the long term (i.e. respectively, about $30 \%$ of reduction for the strength properties and $40 \%$ for the Young's Modulus).

\subsection{Concrete lining degradation}

\subsubsection{Calcium leaching}

This example considers a tunnel excavated in poor-quality sandstones, without a waterproofing system and with concrete weathering due to Calcium leaching. The considered degradation speed of the mechanical properties of the final lining (i.e. Young's Modulus and compressive strength) is similar to the rate of weathering that affects the concrete lining of the Flonzaley tunnel in the Vaud Canton (Switzerland) (Fig. 4). The effect of concrete lining weathering on tunnel equilibrium is represented in the top part of Fig. 8, and the corresponding evolution with time of the 

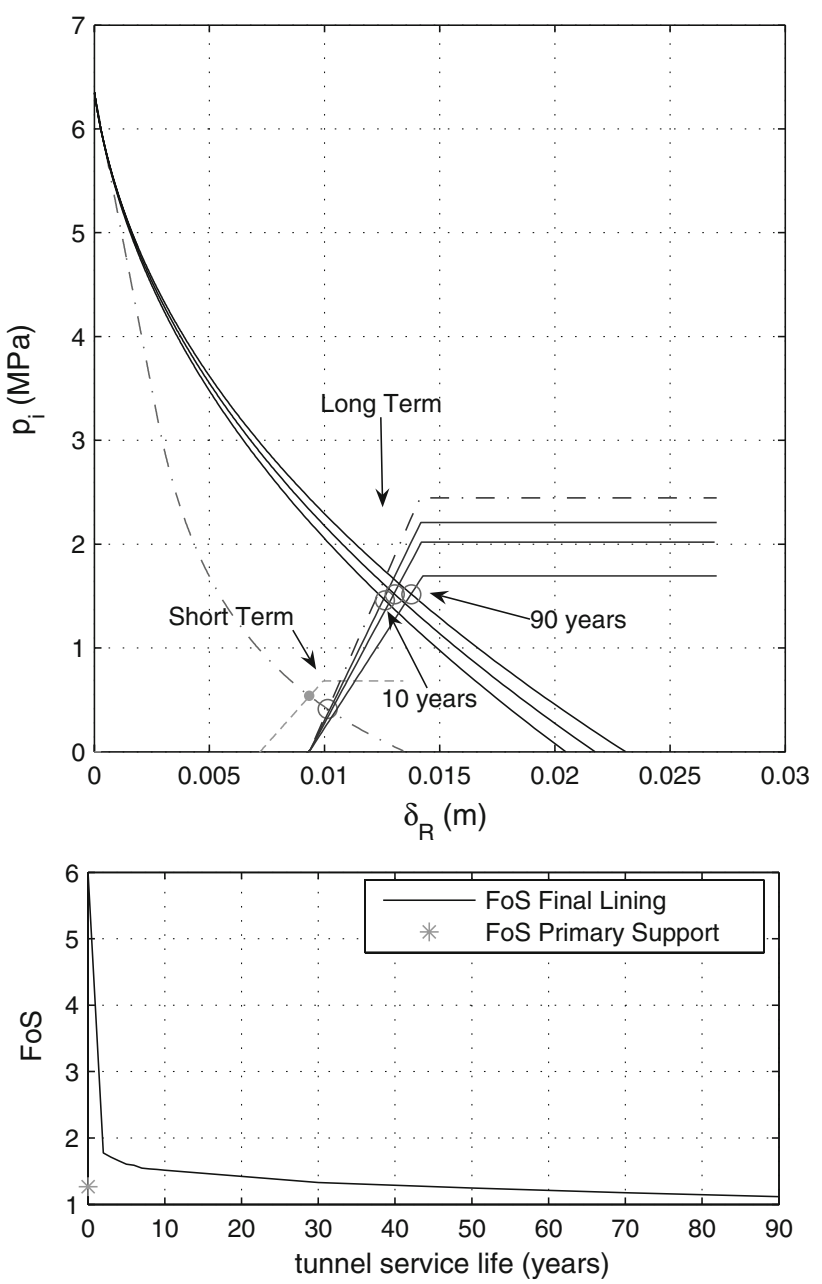

Fig. 10 Top Convergence-confinement analysis for a tunnel with a waterproofing system excavated in good quality marls (see Table 3). Together with ageing of the rock mass (viscoplastic parameters after Boidy et al. 2002), the tunnel is affected by frost de-icing salts attack at tunnel intrados (XF4: splash exposure class). Bottom FoS evolution with time

safety factor is in the lower part. The high tunnel safety factor value in the short term is related to the basic assumption of the convergence-confinement method (axial-symmetry). As this condition is rarely representative of the reality and the convergence-confinement analysis does not allow taking into account the local character of the degradation, this result should be interpreted with particular caution. From a qualitative point of view, however, compared to the rock mass degradations considered in Figs. 6 and 7, the weathering of the final lining appears to develop slower and to affect less significantly the tunnel stability (i. e. slower and smaller decrease of the FoS).

\subsubsection{Corrosion by de-icing salts}

The following example considers a tunnel with a waterproofing system excavated in good-quality marls. No rock mass degradation is considered here. Instead, the internal surface of the final concrete lining is subjected to the deicing salts attack. Considering that the thickness of the weathered zone is proportional to the square root of time (Eq. 10), the rates of the degradation process for the two exposure classes have been chosen based on tunnel inspection observations. In particular:

- For the splash exposure zone (i.e. the lower part of the

side walls), on the left of Fig. 9, it was fixed that $a=$ $5 \mathrm{E}-4\left(\frac{\mathrm{m}}{\sqrt{\text { days }}}\right)$, which corresponds to a thickness reduction of about $50 \mathrm{~mm}$ over 30 years of the tunnel service life.

- For the mist exposure zone (i.e. higher part of the side walls and tunnel crown), on the right of Fig. 9, it was fixed that $a=2.5 \mathrm{E}-4\left(\frac{\mathrm{m}}{\sqrt{\text { days }}}\right)$, which corresponds to a thickness reduction of about $25 \mathrm{~mm}$ over 30 years of tunnel service life.

As in the previous example (i.e. groundwater chemical aggression, Fig. 8), the tunnel safety factor, in the longterm, is affected less by weathering of the final lining compared to rock mass degradation effects (Figs. 6,7). High values of the safety factor are still the consequence of axial-symmetric conditions assumed by the convergence-confinement method. Moreover, due to the fact that the local character of the disorders cannot be taken into consideration in this method, these results should be considered only from a qualitative point of view.

The local changes in the slope of the safety factor's curves are related to the fact that both the stiffness of the final lining and the maximum pressure change due to reduction in thickness. Furthermore, the equilibrium condition depends not only on the final lining stiffness, but also on the slope of the non-linear GCC.

\subsection{Combined effects analysis}

After the separate evaluation of the influence of rock mass and final lining degradations on the long-term behaviour of tunnels, as these processes may happen simultaneously, it is necessary to perform combined analyses. Two examples are proposed in the following:

1. The first describes the evolution of a tunnel, excavated in a rock mass, affected by ageing and with a concrete lining exposed to weathering by de-icing salts attack. The tunnel is provided with a waterproofing system placed at the final lining extrados.

2. The second describes the weathering process of a tunnel without a waterproofing system, excavated in a 
Fig. 11 Top Convergenceconfinement analysis for a tunnel without a waterproofing system excavated in poor quality sandstones (see Table 3), considering weathering effects on both the rock mass (i.e. reduction in the strength properties of $30 \%$ of their initial values (Eq. 3) and the concrete lining (i.e. reduction in the mechanical properties due to aggressive groundwater leaching (Eqs. 8, 9, 10). The GCC in crown (dashed curve) takes into account the roof effects (i.e. the loss of confinement of the excavated rock above the tunnel crown). Bottom FoS evolution with time, respectively, at tunnel crown and side walls
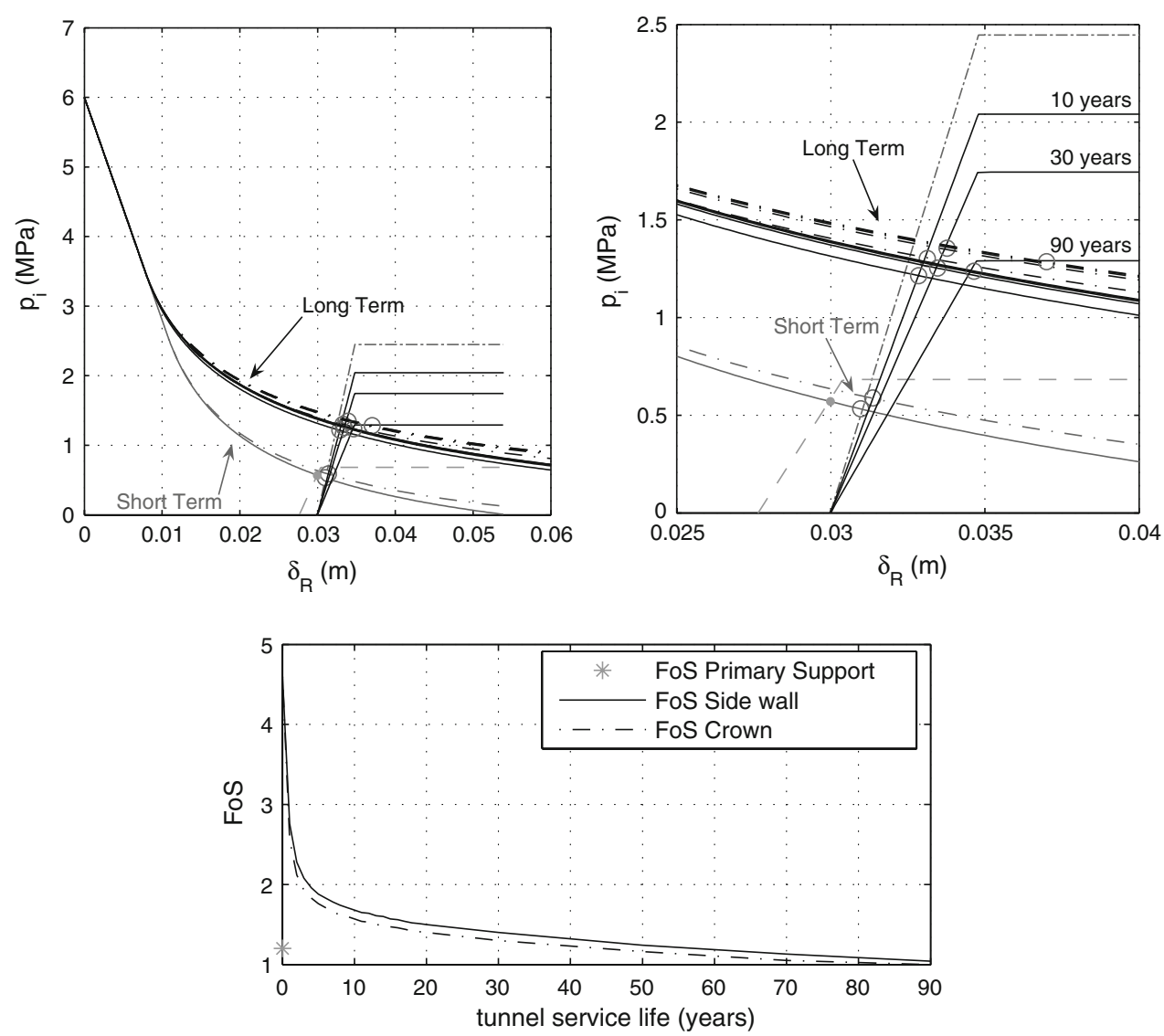

Table 3 Mechanical parameters for two typical Swiss rock formations

\begin{tabular}{|c|c|}
\hline & GCC Short Term Conditions \\
\hline $\begin{array}{l}\text { Poor quality sandstones } \\
\gamma_{m}=24 \frac{\mathrm{kN}}{\mathrm{m}^{3}} \\
E=2,000 \mathrm{MPa} \\
\nu=0.25 \\
\varphi=30^{\circ} \\
c=0.5 \mathrm{MPa}\end{array}$ & $0_{0}^{0.02}$\begin{tabular}{c|}
0.04 \\
$\delta_{R}(m)$
\end{tabular} \\
\hline $\begin{array}{l}\text { Good quality marls } \\
\gamma_{m}=25.4 \frac{\mathrm{kN}}{\mathrm{m}^{3}} \\
E=8,000 \mathrm{MPa}^{\mathrm{a}} \\
\nu=0.4 \\
\varphi=20^{\circ} \\
c=1 \mathrm{MPa}\end{array}$ & $\begin{array}{l}{ }_{0}^{0.01} \\
\delta_{\delta_{R}(m)} \\
0.02\end{array}$ \\
\hline
\end{tabular}

a This value, though it may seem quite high for common marls (usually characterised by smaller values, i.e. $E \sim 3,000 \mathrm{MPa}$ ), is valid for silty-marls of the Jura (Boidy et al. 2002)

rock mass with a high weathering potential and affected by aggressive groundwater inflow (concrete lining deterioration, e.g. Calcium leaching).

\subsubsection{Rock mass ageing and concrete lining corrosion}

This example considers a tunnel excavated in good quality marls with a waterproofing membrane at the final lining extrados. In this case, the deterioration of the final lining develops at the intrados and is due to projection of de-icing salts caused by rolling traffic. It has been modelled by a thickness reduction for a splash exposure class as described in Sect. 4.2.2. Moreover, an ageing of the marls is considered according to Sect. 4.1.1. By combining the effects of the reduction in the final lining thickness and the increasing external load, the safety factor may significantly decrease in the long term, as shown in Fig. 10. Though the weathering of concrete lining has a reduced influence on the long-term tunnel equilibrium, it is important to observe that, together with rock mass ageing, without a regular maintenance of the structure, it may cause some problems.

\subsubsection{Weathering of rock mass and concrete lining}

If a tunnel is excavated in a sedimentary rock, in the long term it may show symptoms of rock mass weathering. Moreover, when waterproofing system is absent or does not work properly, the concrete lining may be affected in the long term by aggressive groundwater weathering action. In 
this case, both the convergence and the confinement curves change in the long term, modifying tunnel equilibrium conditions. Figure 11 shows the long-term convergence and confinement curves for a tunnel without a waterproofing membrane, excavated in poor quality sandstones (see Table 3). The rock mass and final lining weathering processes have been modelled as follows:

- rock mass strength properties were reduced by $30 \%$ according to the hyperbolic law expressed by Eq. 3 (Sect. 3.2.2), with $T$ equal to 1 year;

- concrete lining mechanical properties were reduced as described in Sect. 3.3.1, with a weathering rate equal to $a=5.2 \mathrm{E}-4\left(\frac{\mathrm{m}}{\sqrt{\text { days }}}\right)$.

Due to the rock mass weathering, and to the consequent loss of confinement of the zone above the tunnel crown, the axisymmetric condition is no longer satisfied and two GCCs are represented, respectively, for side walls (i.e. solid curves) and crown (i.e. dashed curves). As already observed in Sect. 4.1.2, the difference between the curves of the crown and side walls is low. Thus, the safety factor was evaluated still under the hypothesis of axisymmetric loads, respectively, for crown and side walls (Fig. 11, bottom).

By comparing the short-term and the long-term conditions, it is possible to observe an important reduction in the tunnel safety factor already after 10 years of service life. This is mainly due to the rock mass weathering effects. Then, the reduction is slower, mainly determined by the weathering of the concrete lining process. Moreover, in this case a failure may be expected before the end of the service life.

\section{Results and discussion: a methodology for the evaluation of tunnel conditions}

After construction, the tunnel equilibrium is modified due to interactions with the environment together with operational conditions. Moreover, due to the rheological behaviour of the rock mass and the final lining, stresses and strains may change. Although limited by restrictive assumptions, mainly due to its simplicity and widespread use, the convergence-confinement approach has been chosen in this framework for describing, both separately and in a combined way, the influence of rock mass and final lining degradations on the change in tunnel conditions. The effects of pathologies have been modelled by modifying mechanical and/or geometrical properties characterising concrete lining and rock mass. Long-term pressures and displacements modified by degradation processes have been evaluated by integrating these models into the basic equations of the characteristic lines. The evolution of equilibrium conditions during the tunnel service life has been interpreted by means of a safety factor for the final lining structure.

By considering separately rock mass and final lining degradations, an interesting result is that lining degradation seems to affect less significantly the long-term stability of the tunnel than rock mass degradation. Nonetheless, the influence of the final lining degradation might have been much more important if the local character of lining pathologies could have been taken into consideration. It is obvious that the consequences in the long term are worse if the rock mass and the final lining degradations are superposed. A good estimation of the rate of rock mass degradation is necessary for a better assessment of long-term stability conditions of the tunnel. Actually, by changing the viscoplastic parameters in Eq. 2 or the value of the constant $T$ in Eq. 3 (i.e. by reducing the rate of the rock mass degradation processes), the evolution of the tunnel safety factor may be more influenced by the final lining degradation.

For improving long-term tunnel stability assessment, the results show also the importance of taking into account all of the construction details. For example, the origin of the confinement line, which represents the radial displacement already undergone by the walls when the support is installed and which has been evaluated using the similarity principle introduced by Corbetta (1990), has clearly a major influence on the equilibrium state. On the other hand, as far as the tunnel primary support and final lining are concerned, it is clear that the perfect axial-symmetric condition assumed by the convergence-confinement method induces an overestimation of the safety factor, at least at the beginning of the tunnel service life. Actually, as observed for the majority of the Swiss National Road tunnels, axisymmetric loads and perfect circular concrete linings are rarely representative of reality, i.e.:

1. in very few cases the tunnel has a perfect circular shape;

2. the loading conditions do not respect the axialsymmetric assumption except for very deep tunnels;

3. many disorders and pathologies have a local character (i.e. are limited to some tunnel parts).

As a consequence of those restrictive assumptions, the convergence-confinement method should be seen as a tool that helps to understand and roughly estimate the change in tunnel equilibrium conditions. Nonetheless, the basic idea that the equilibrium of a tunnel results from a rock-support/ lining interaction and that both components can evolve with time should be considered in any evaluation of tunnel stability and serviceability. Inspired by this, Fig. 12 presents a general methodology that considers the initial conditions of the tunnel (function of the rock mass, the 


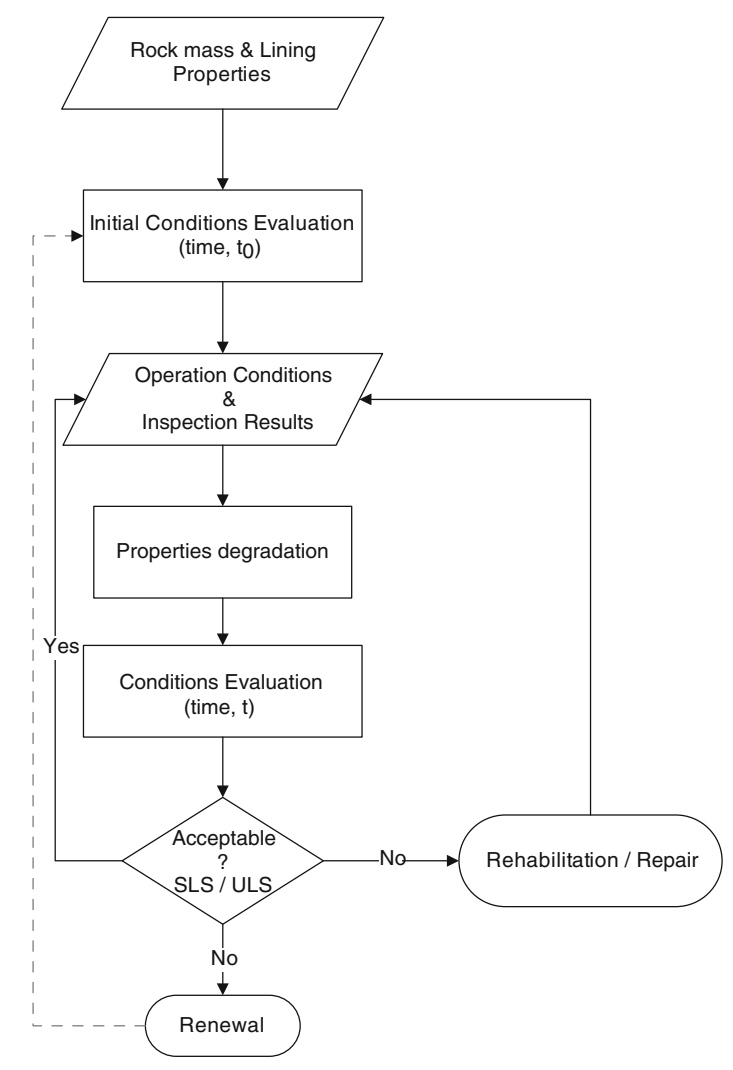

Fig. 12 Methodology for evaluating long-term tunnel equilibrium condition ( $S L S$ serviceability limit state, $U L S$ ultimate limit state)

supporting structure and the construction process) and their evolution during service life due to degradation processes in both the rock mass and the final lining (function of the environment and the operational conditions).

Degradation symptoms, caused by both rock mass and final lining pathologies, appear in the form of disorders and damages affecting visible parts of the tunnel (i.e. final lining intrados, drainage system, gutter, ventilation slab, walls foundations and road track) and may be observed during principal inspections. All information related to tunnel operation conditions and observed disorders should be used for updating initial data and re-evaluating the tunnel equilibrium at regular intervals during its service life. In order to choose appropriate conservation measures, the new tunnel conditions should be analysed in terms of serviceability and ultimate limit state (i.e. respectively, SLS and ULS).

In addition to a qualitative assessment of tunnel conditions from inspection observations (i.e. quality indexes), a more quantitative evaluation could be adopted, e.g. by means of more refined numerical analyses (FEM), which could in particular take into account the local effects of some degradation processes. The general evaluation methodology presented in Fig. 12 may be applied to both qualitative and quantitative approaches.

\section{Conclusions}

A better understanding of the evolution with time of the conditions of road tunnels is necessary for improving management and conservation practices. This paper shows how the delayed behaviours of final lining and rock mass influence long-term stability conditions of the tunnel.

Tunnel durability is usually affected by complex interactions between rock mass, groundwater and concrete lining, as well as traffic and operational and environmental conditions inside the tunnel, together with conservation procedures during service life. Starting from the initial tunnel conditions (e.g. rock mass properties and behaviour; tunnel geometry, construction method; supporting structure and behaviour), it is necessary to identify the main degradation processes and to model them for estimating their effects on the long-term tunnel conditions.

Starting from the main pathologies affecting Swiss National Road tunnels, degradation effects of both the rock mass and concrete lining have been modelled in the framework of the convergence-confinement method. The separate influence of the rock mass and the final lining degradations has been analysed first, followed by two combined examples. The results (i.e. changing tunnel equilibrium) have been interpreted by evaluating the decrease in safety factor with time during the tunnel service life.

Though the basic assumptions of the convergenceconfinement method are rarely met in real cases, some important observations can be pointed out:

1. The long-term stability assessment of tunnels requires first a correct appraisal of the short-term equilibrium, ensured by the primary support during construction, and of the medium-term conditions (few years later) when the rock load has been transferred from the primary support to the final lining.

2. Then, in order to verify the long-term equilibrium of a tunnel, it is necessary to take into consideration the delayed behaviour of both the excavated rock mass and the final lining. The effects of the main degradation processes can be modelled in a simple way by changing the mechanical and geometrical features of the tunnel.

3. A detailed characterisation of the tunnel and the surrounding rock mass as well as a constant update of these data are necessary in order to perform a complete diagnostic of the degradation processes and to estimate their contribution to modifying long-term conditions.

4. The relative influence of the rock mass and the final lining degradations on the long-term tunnel conditions is a function of the importance and rate of the processes. In the considered examples, the delayed 
behaviour of the rock mass seems to be more significant and faster than the final lining degradation. As a result, the evolution of the safety factor appears to be mainly influenced by the degradation of the rock mass in the medium term, and of the lining in the long term.

Due to the local character of several disorders and to the major restrictions of the convergence-confinement method (circular tunnel and axisymmetric in situ stress conditions), the proposed approach should be considered as a first tool to understand and roughly estimate the influence of degradations on tunnel conditions. Nonetheless, the basic idea that the equilibrium of a tunnel results from a rock-supporting structure interaction and that both components evolve with time should be considered in any evaluation of tunnel stability and serviceability. Inspired by this, a general methodology is proposed. After the evaluation of the initial equilibrium, all information related to the tunnel operation conditions and disorders observed during principal inspections should be used for updating initial data and re-evaluating the tunnel equilibrium at regular intervals during its service life. It is worthwhile to observe that this methodology for evaluating long-term conditions of the tunnel can be applied to both qualitative and quantitative approaches. In particular, it could be used with numerical analysis (FEM) for representing also the local character of identified disorders and thus refining the results.

Acknowledgments The authors thank the Swiss Federal Road Authority, OFROU for the financial support (grant no. FGU2003/ 002). They also thank Ruth Harding for having carefully checked the English in the manuscript.

\section{References}

Barla G (2001) Tunnelling under squeezing rock conditions. In: Kolymbas D (ed) Tunnelling mechanics. Advances in Geotechnical Engineering and Tunnelling, vol 5. Eurosummerschool, Innsbruck, pp 169-268

Berest P, Nguyen Minh D (1983) Modèle viscoplastique pour le comportement d'un tunnel revêtu. Rev Fr Géotech 24:19-25

Boidy E (2002) Modélisation numérique du comportement différé des cavités souterraines. Thèse de doctorat, Université Joseph Fourier - Grenoble I, Grenoble

Boidy E et al (2002) Back analysis of time-dependent behaviour of a test gallery in claystone. Tunn Undergr Space Technol 17(4):415-424

Brady BHG, Brown ET (2004) Rock mechanics for underground mining. Kluwer Academic Publishers, Dordrecht/Boston/London

Brown ET et al (1983) Ground response curves for rock tunnels. J Geotech Eng 109:15-39

Bultel F (2001) Prise en compte du gonflement des terrains pour le dimensionnement des revêtements des tunnels. Thèse de doctorat, Ecole Nationale des Ponts et des Chaussées, Paris

Caquot A, Kerisel J (1956) Traité de mécanique des sols. GauthierVillars, Paris
Carde C, Francois R (1997) Effect of the leaching of calcium hydroxide from cement paste on mechanical and physical properties. Cement Concrete Res 27(4):539-550

Carranza-Torres C, Fairhurst C (2000) Application of the convergence-confinement method of tunnel design to rock-masses that satisfy the Hoek-Brown failure criterion. Tunn Undergr Space Technol 15(2):187-213

Carranza-Torres C, Zhao J (2008) Analytical and numerical study of the effect of water pressure on the mechanical response of cylindrical lined tunnels in elastic and elasto-plastic porous media. Int J Rock Mech Min Sci. doi:10.1016/j/ijrmms.2008. 09.009

CEB (1993) CEB-FIP Model Code 1990. Thomas Telford, London

CETu (2004) Guide de l'inspection du génie civil des tunnels routiers. Des désordres vers lediagnostic, CETu, France

Constantinescu M, Cristescu N (1983) Creep of rock-like materials. Int J Eng Sci 1:45-49

Corbetta F (1990) Nouvelles méthodes d'étude des tunnels profonds. Calculs analytiques et numériques. Thèse de doctorat, École Nationale Superiore des Mines de Paris, Paris

Cristescu N (1985) Viscoplastic creep of rocks around horizontal tunnels. Int J Rock Mech Min Sci Geomech Abstr 22(6):453-459

Cristescu N (1988) Viscoplastic creep of rocks around a lined tunnel. Int J Plast 4(4):393-412

Cristescu N (1994) Time effect in rocks surrounding a horizontal tunnel. In: Nelson PP, Laubach SE (eds) Proceedings of the 1st NARMS Symposium Rock Mechanics. Models and measurements. Challenges from industry. Balkema, Rotterdam, pp 657-664

Cristescu N, Hunsche U (1998) Time effects in rock mechanics. Wiley, West Sussex

Daemen JJK (1975) Rational design of tunnel supports: tunnel support loading caused by rock failure. Ph.D. thesis, University of Minnesota, Minneapolis

Fritz P (1984) An analytical solution for axisymmetric tunnel problems in elastoviscoplastic media. Int J Numer Anal Methods Geomech 8:325-342

Gärber R (2003) Design of deep galleries in low permeable saturated porous media. Thèse EPFL no. 2721, École Polytechnique Fédérale de Lausanne, Lausanne

Grobbelaar C (1994) The degradation and failure of concrete linings around water conveyance tunnels. Tunn Undergr Space Technol 9(1):67-71

Gysel M (1987) Design of tunnels in swelling rock. Rock Mech Rock Eng 20(4):219-242

Hagros A, Johansson E, Hudson JA (2007) Time dependency in the mechanical properties of crystalline rocks: a literature survey. Posiva Working Report, Finland

Kaufmann J (2000) Experimental identification of damage mechanisms in cementitious porous materials on phase transition of pore solution under frost deicing salt attack. Thèse EPFL no. 2037, École Polytechnique Fédérale de Lausanne, Lausanne

Ladanyi B (1974) Use of the long-term strength concept in the determination of ground pressure on tunnel linings. In: Advances in rock mechanics: reports of current research, Proceedings of the 3rd international congress on rock mechanics. National Academy of Sciences, Denver, pp 1150-1156

Ladanyi B (1980) Direct determination of ground pressure on tunnel lining in a non-linear viscoelastic rock. In: Proceedings of the 13th Canadian rock mechanics symposium. CIM, Montreal, pp $126-132$

Nguyen V-H (2005) Couplage dégradation chimique-comportement en compression du béton. Thèse de doctorat, Ecole Nationale des Ponts et Chaussées, Paris

Nguyen Minh D, Pouya A (1992) Une méthode d'étude des excavations souterraines en milieu viscoplastique. Prise en 
compte d'un état stationnaire des contraintes. Rev Fr Géotech 59:5-14

Oreste P (2003) A procedure for determining the reaction curve of shotcrete lining considering transient conditions. Rock Mech Rock Eng 36(3):209-236

Oreste P, Peila D (1997) Modelling progressive hardening of shotcrete in convergence-confinement approach to tunnel design. Tunn Undergr Space Technol 12(3):425-431

Pacher F (1964) Deformationmessungen in Versuchsstollen als Mittel zur Erforschung des Gebirgsverhaltens und zur Bemessung des Ausbaues. Felsmech Ingenieursgeol Suppl IV:149-161

Panet M (1979) Time-dependent deformations in underground works. In: Proceedings of the 4th international congress on rock mechanics, Montreux, 2-8 Sept 1979. Balkema, Rotterdam, pp 279-290

Panet M (1995) Le calcul des tunnels par la méthode convergenceconfinement. Presses d'Ecole Nationale des Ponts et Chaussées, Paris

Park K-H, Kim Y-J (2006) Analytical solution for a circular opening in an elastic-brittle-plastic rock. Int $\mathrm{J}$ Rock Mech Min Sci 43(4):616-622

Rousset G (1990) Les sollicitations à long terme des revêtements des tunnels. Rev Fr Géotech 53:5-20
Sandrone F (2008) Analysis of pathologies and long term behaviour of Swiss National Road tunnels. Thèse EPFL no. 4019, École Polytechnique Fédérale de Lausanne, Lausanne

Sandrone F et al (2007) Data collection for Swiss Road tunnels maintenance. Felsbau 1:8-14

SIA 197 (2004) Projets de tunnels. Bases générales, SIA, Zurich

SN EN 206-1 (2000) Béton-Partie 1: Spécification, performances, production et conformité. SIA, Zurich

Sulem J (1994) Analytical methods for the study of tunnel deformation during excavation. In: Barla $\mathrm{G}$ (ed) Gallerie in condizioni difficili MIR'94, Torino, pp 301-317

Sulem J et al (1987) An analytical solution for time-dependent displacements in a circular tunnel. Int $\mathbf{J}$ Rock Mech Min Sci Geomech Abstr 24(3):155-164

Széchy K (1966) The art of tunnelling. Akadémiai Kiadó, Budapest

Terzaghi K (1946) Rock defects and loads on tunnel supports. Commercial Shearing and Stamping Company, Youngstown

Wang $\mathrm{K}$ et al (2006) Damaging effects of deicing chemicals on concrete materials. Cement Concrete Compos 28(2):173-188

Yokozeki K et al (2004) Modeling of leaching from cementitious materials used in underground environment. Appl Clay Sci 26(1-4):293-308 